\title{
Conceitos de automação na medicina laboratorial: revisão de literatura
}

\author{
Concepts in laboratory medicine automation: literature review
}

Gustavo Aguiar Campana'; Carmen Paz Oplustil ${ }^{2}$

unitermos
Medicina laboratorial
Automação
Processos
Tendências

\section{resumo}

Nas últimas décadas, a introdução da automação na medicina laboratorial foi destacada como a espinha dorsal na busca de eficiência e viabilidade das empresas atuantes nesse setor e expandiu-se em todas as fases dos processos no laboratório clínico: pré-analítica, analítica e pós-analítica. A implementação de um processo de automação laboratorial deve levar em consideração o posicionamento estratégico da empresa e sua forma de atuação. Diferentes modelos de processos automatizados funcionam para diferentes negócios, definidos pelo mix de exames, volume de processamento, atributos estratégicos necessários, capacidade de investimento, entre outros. Este artigo tem como principal objetivo apresentar uma breve revisão dos processos automatizados disponíveis em medicina laboratorial.

\section{abstract}

In the last decades, the introduction of automation in laboratory medicine has played a major role in the search for efficiency and viability promoted by enterprises from this sector. Additionally, it has been expanded to all phases and processes within clinical laboratories: pre-analytical, analytical and post-analytical. Automation program implementation must take into account the company's strategic planning and business approach. Different automated processes cater for different business platforms, which are defined by test mix, workflow volume, required strategic characteristics, investment capacity, among others. This article aims at briefly reviewing the automation processes available in laboratory medicine. key words

Laboratory medicine

Automation

Workflow

Trends

1. Patologista clínico; sócio-diretor da Formato Clínico.

2. Biomédica; mestre em Microbiologia; sócia-diretora da Formato Clínico. 


\section{Introdução}

Este artigo tem como principal objetivo apresentar uma breve revisão dos processos automatizados disponíveis em medicina laboratorial. As vantagens e desvantagens de cada modelo existente dependem exclusivamente dos objetivos e das estratégias de cada empresa que implementa um processo como este.

\section{Definição}

O avanço da tecnologia médica e seu impacto na assistência à saúde e na economia desta são pautas de discussão de diversas frentes atuantes na área.

A palavra automação é proveniente do latim automatus e significa mover-se por si. Diversas definições estão disponíveis, mas, em resumo, automação é a aplicação de técnicas computadorizadas ou mecânicas com o objetivo de tornar um processo mais eficiente, maximizando a produção com menor gasto de energia e gerando maior segurança. Entendemos por gasto de energia a aplicação de mão de obra especializada em atividades de baixa geração de valor, gasto de tempo, desperdícios etc.

Nas últimas décadas, a introdução da automação na medicina laboratorial foi destacada como a espinha dorsal na busca de eficiência e viabilidade das empresas atuantes nesse setor e expandiu-se para todas as fases dos processos no laboratório clínico (pré-analítica, analítica e pós-analítica), além da tecnologia e de sua evolução permitirem que empresas de diferentes portes e quantidades de exames implementassem algum tipo de automação em seus processos ${ }^{(10)}$

Hoje, podemos considerar a automação como um processo customizado que varia da automação de uma etapa do processo analítico até a automação total do laboratório e que gera para o mercado uma proliferação de boas práticas por meio de padronizações ${ }^{(9)}$.

\section{Automatizar? Motivos e expectativas}

Diferentes fatores serviram de motivadores para a rápida evolução da automação na medicina laboratorial: fatores externos de mercado, necessidade de melhor assistência à saúde e fatores internos de operação e do ambiente financeiro econômico das instituições.
Em termos de mercado, os fatores motivadores são traduzidos pela exigência de altos padrões de qualidade e pelas expectativas do cliente referentes aos serviços prestados. No âmbito assistencial, a segurança do paciente por meio da minimização de erros e a redução dos prazos de resultados são fatores de impacto positivo no manejo dos pacientes e, consequentemente, na economia da saúde. Ressaltamos que a imensa pressão exercida na redução dos custos e a busca por mais produtividade, viabilizando empresas a permanecerem no $\operatorname{mercado}^{(9)}$, são dois dos principais motivos da procura por automação de processos.

A necessidade constante da busca pela otimização dos custos nos laboratórios clínicos tem sua base na redução dos valores médios dos exames. Nos EUA, por exemplo, a receita média por exame em 1990 era de US\$24; em 1995, US\$16; em 2005, próximo a US\$10. Nesse contexto, a distribuição dos custos técnicos sofreu uma mudança importante. Em 1991, os gastos foram representados por $43 \% \mathrm{em}$ quadro pessoal, $35 \%$ em equipamentos e reagentes e $22 \%$ de despesas gerais; enquanto em 1999 essa distribuição foi de $65 \%$, $15 \%$ e $20 \%$, respectivamente. Essa tentativa de diminuir os valores gastos junto às indústrias de diagnóstico in vitro demonstra a pressão por reduções de custos sendo transmitida por toda a cadeia ${ }^{(8)}$.

Focando na operação, destacamos como fatores internos de pressão os relacionados com os colaboradores (maior segurança e possibilidade de deslocamentos verticais e horizontais para possíveis expansões e outras atividades $)^{(9)} \mathrm{e}$ aqueles diretamente associados a planejamento da capacidade de produção e throughput (produtividade do processo), por meio da eliminação de atividades desnecessárias e da combinação das atividades em processos únicos, buscando a simplificação $0^{(10)}$ e a padronização do processo, o que leva à redução na quantidade de documentação de suas atividades ${ }^{(13)}$. A automação na medicina laboratorial também permite o gerenciamento remoto dos analisadores, a padronização de protocolos de interfaceamento mecânico e eletrônico e a facilidade na integração de plataformas ${ }^{(4)}$.

Em relação às expectativas em um processo de automação, além de alcançar os objetivos que motivaram a busca por esse tipo de processo, estão as necessidades operacionais que devem ser atingidas, com foco principal na viabilização da empresa e não em torná-la algo extremamente amarrado e inerte. A Tabela 1 traz um resumo das principais expectativas na implementação de um processo automatizado. 


\section{Tabela 1 Expectativas do processo de automação laboratorial ${ }^{(9)}$}

\begin{tabular}{|c|c|}
\hline Categoria & Expectativas \\
\hline Metodologia & $\begin{array}{l}\text { - Absorver os testes atuais com possibilidade de canais abertos } \\
\text { - Performance dos ensaios aceitável } \\
\text { - Ensaios com aprovação pelos órgãos vigentes } \\
\text { - Tempo de manutenção aceitável }\end{array}$ \\
\hline TAT & $\begin{array}{l}\text { - TAT de } 30 \text { minutos para bioquímica e } 40 \text { minutos para imunoensaios nos testes } \\
\text { de urgência (STAT) } \\
\text { - Habilidade do sistema para priorização de amostras (modo STAT) }\end{array}$ \\
\hline Manuseio de amostras & $\begin{array}{l}\text { - Diminuição dos processos manuais e erros do operador } \\
\text { - Possibilidade de utilização de diferentes tipos de tubos } \\
\text { - Presença de alertas de baixo volume, hemólise, lipemia, fibrina etc. } \\
\text { - Equipamentos analíticos com possibilidade de operação em caso de parada da } \\
\text { linha }\end{array}$ \\
\hline $\begin{array}{l}\text { Throughput } \\
\text { (produtividade do processo) }\end{array}$ & $\begin{array}{l}\text { - A capacidade máxima deve ser capaz de absorver o crescimento na quantidade de } \\
\text { exames }\end{array}$ \\
\hline Investimento & - Capacidade de retorno do valor investido devido aos benefícios financeiros gerados \\
\hline Tecnologia da informação & $\begin{array}{l}\text { - Interfaces diretas e bidirecionais } \\
\text { - Capacidade de manter-se em operação caso haja problemas em sistemas } \\
\text { interfaceados (p. ex.: HIS) } \\
\text { - Fornecer processos de autovalidação de resultados e programas de controle de } \\
\text { qualidade } \\
\text { - Fornecer e gerenciar o armazenamento das informações }\end{array}$ \\
\hline Ambiente e segurança & $\begin{array}{l}\text { - A instalação não deve prejudicar o ambiente de trabalho (geração de calor, ruídos, } \\
\text { - Minidade etc.) } \\
\text { - Mecessidade mínimar riscos de exposição ocupacional } \\
\text { - Nequações de espaço disponível }\end{array}$ \\
\hline Disponibilidade e manutenção & $\begin{array}{l}\text { - Os períodos de parada devem ser mínimos (downtime) } \\
\text { - Possibilidade de assistência técnica local } \\
\text { - Disponibilidade de assistência } 24 \text { horas e } 365 \text { dias }\end{array}$ \\
\hline
\end{tabular}

TAT: tempo de atendimento total; STAT: tempo de disponibilização de resultados reduzido.

\section{Fluxo operacional no laboratório e possibilidades de automação}

As etapas do fluxo dos processos em um laboratório de patologia clínica já são bem definidas e compreendem as fases pré-analítica, analítica e pós-analítica.

\section{Fase pré-analítica}

Os processos anteriores à realização do ensaio laboratorial propriamente dito (fase pré-analítica) são componentes fundamentais da qualidade dos serviços do laboratório. Essa etapa compreende a coleta, a manipulação, o processamento e a entrega das amostras aos analisadores.

$\mathrm{Na}$ literatura científica, observamos que essa fase absorve o maior número de erros cometidos nos laboratórios clínicos, variando de $31,6 \%$ a $84,5 \%$ de todos os erros laboratoriais ${ }^{(1,3)}$, demonstrando a importância dos processos automatizados nessa etapa.

Neste artigo, denominaremos linha o processo de transporte automatizado das amostras até seu destino final, 
seja ele o equipamento analítico ou o módulo de armazenamento. Diferentes modelos de automação das linhas já foram implementados em diversos laboratórios e discutidos na literatura. Citaremos três tipos(4).

- Robô móvel: modelo no qual o transporte ocorre por um robô que se movimenta até os equipamentos. Esses modelos são geralmente de fácil adaptação a diferentes tipos de amostras e tamanhos de tubos, além de flexíveis quanto às mudanças de layout das áreas técnicas. As principais desvantagens nesse modelo de automação são o transporte em batelada, com impacto no throughput e, consequentemente, no tempo de atendimento total (TAT), e a dificuldade de uma interface mecânica entre o robô e o equipamento analítico, necessitando, muitas vezes, de uma ação manual(4).

- Esteiras de transporte: a automação por esteiras de transporte foi bastante difundida na prática da medicina laboratorial. Suas principais desvantagens estão relacionadas com pequena flexibilidade de tipos de amostras a serem transportadas e dificuldade de alteração de layout ou introdução e expansão com novos equipamentos. A interface da esteira com os equipamentos pode ocorrer de forma direta (denominado point-in-space) ou por meio de braços mecânicos ${ }^{(4)}$. Como principais vantagens, temos a garantia de um fluxo contínuo totalmente automatizado, com mais benefícios na integração de metodologias e compartilhamento de analisadores.

- Braços robóticos: são braços articulados que podem se movimentar em diferentes direções. De todos os tipos existentes, dois se destacam nas aplicações em medicina laboratorial: cilíndricos e articulados. A aplicação desses modelos difere na dependência da configuração dos equipamentos no entorno (como celular ou linear), na base de instalação do braço (móvel ou estacionária) e nas articulações presentes nos braços (como articulações tipo cotovelo e joelho).

- Os braços robóticos apresentam boa reprodutibilidade dos movimentos, porém necessitam de conhecimento específico da equipe na introdução de novos protocolos em momentos de mudanças como expansão e novas máquinas ${ }^{(4)}$.

As principais considerações nos processos de automação nessa etapa são(9):

- configuração da linha - compreende a disposição dos equipamentos analíticos e sua relação com a linha (forma paralela ou perpendicular) e o desenho dela (linear ou circular). Os fatores que são impactados pelas considerações de configuração são relacionados principalmente com o espaço físico e o tempo gasto para repetições de exames, diluições e testes reflexos, visto que, dependendo da conformação da linha, existem formações de filas de amostras, além da necessidade de fácil acesso aos equipamentos analíticos para manutenções e operação manual quando necessário;

- transporte de amostras na linha - os sistemas automatizados possuem transporte direto do tubo (transporte simples) ou por meio de racks (transporte múltiplo) geralmente de cinco amostras. Em geral, o sistema múltiplo de transporte possui maior velocidade, porém é preciso ter convergência entre a informação presente nas amostras e aquela presente na própria rack, além da necessidade, dependendo da configuração, da permanência das cinco amostras na linha quando uma delas precisar de repetição ou diluição;

- tipos de tubo - diferentes tamanhos e tipos de tubos estão disponíveis no mercado. A utilização de um ou outro depende, muitas vezes, da área de atuação do laboratório. Por exemplo, laboratórios de apoio ou referência recebem amostras em um número variado de tipos de tubos; sua padronização é difícil e reduz a flexibilidade no atendimento ao cliente;

- identificação da amostra - código de barras e radiofrequência (RFID) são duas principais formas de identificação disponíveis. Deve-se considerar a sensibilidade dos leitores, a presença de umidade e os atritos mecânicos que são passiveis de ocorrer;

- entrega e seleção de amostras - os sistemas devem comportar amostras provenientes de alíquotas, tubos primários ou ambos. Essa análise deve sempre levar em consideração o mercado de atuação do laboratório e o fluxo dos processos no mesmo. $\mathrm{Na}$ seleção das amostras, metodologias de detecção dos índices séricos (hemólise, lipemia e icterícia), volume da amostra e presença de fibrina devem estar presentes e serem assinalados de alguma forma (flag);

- centrifugação - o processo de centrifugação das amostras deve ser analisado em sua capacidade (número de amostras e tempo de centrifugação) e funcionalidade (tipos de tubo, temperatura e rotação). Deve-se considerar o número de centrífugas 
presentes na linha, principalmente para laboratórios de grande volume ou que utilizarão a mesma linha para amostras de urgência (STAT) ou para ensaios de coagulação (necessidade de rotação e velocidade maiores);

- decapping/recapping - as atividades de destampar os tubos para análise e posterior fechamento das amostras para armazenagem devem ser alinhadas aos tipos de tubo que serão utilizados na linha. Os dois principais modelos de fechamento de amostras são por meio de tampas plásticas ou por selamento com metal aquecido.

\section{Fase analítica}

A maior evolução da tecnologia na medicina laboratorial ocorreu na etapa analítica. Hoje, podemos considerar que, vistos de forma isolada, praticamente todos os equipamentos analíticos são processos automatizados.

A maioria dos equipamentos analíticos iniciou com desenhos de ensaios em cuvetas, geralmente dispostos em um fluxo contínuo, provenientes principalmente da automação dos ensaios bioquímicos enzimáticos. A necessidade da implementação de diferentes detectores de sinais e da adsorção de moléculas em fases sólidas trouxe ao mercado novas gerações de equipamentos, principalmente para diferentes metodologias imunológicas ${ }^{(2)}$.

As principais considerações na definição das plataformas analíticas e suas conformações devem levar em consideração as plataformas analíticas e a configuração.

\section{Plataformas analíticas}

A definição dos equipamentos a serem utilizados deve estar sempre alinhada ao tipo de automação a ser implementada. Os principais parâmetros a serem determinados são referentes a capacidade e velocidade de produção, throughput, tempo e necessidade de manutenção, modo de utilização de controles e estabilidade das calibrações, metodologias disponíveis e preparação de reagentes.

A quantidade (tipos) de reagentes que podem abastecer os equipamentos (denominada on board) reduzindo os períodos de máquinas paradas para reabastecimento e calibrações tem impacto significativo no processo de automação e em sua capacidade de produção e throughput.

Nos últimos anos, algumas das principais tendências em automação laboratorial foram a introdução dos conceitos de consolidação, ou seja, uma pressão de convergência de mais tipos de exames em uma única plataforma, e a integração, focada na obtenção de plataformas únicas com diferentes metodologias, como, por exemplo, a integração de imunoensaios com ensaios bioquímicos enzimáticos. Em ambos os conceitos, diversos benefícios foram gerados ao processo do laboratório, como a possibilidade de operação com menos tubos (muitas vezes, um único), diminuição do TAT, maior produtividade operacional, diluição de custos fixos, entre outros.

A disposição das plataformas analíticas deve ser um ponto de atenção quanto a sua ordem, ou seja, relação entre velocidade, capacidade de produção e throughput e possibilidade de carregamento de interferentes entre analitos (carryover). Por exemplo, os ensaios de bioquímica clínica são, em sua maioria, de alta velocidade e utilizam metodologias colorimétricas enzimáticas, de sensibilidade analítica na ordem de três a 10 vezes menor que os imunoensaios (p. ex., quimioluminescência), que acabam por aumentar a possibilidade de interferência ${ }^{(2)}$. Outro importante aspecto a ser considerado é o alinhamento ao modelo de negócio da empresa com as plataformas a serem instaladas e os tipos de exames (menu) disponíveis. Laboratórios que atuam em mercados de apoio ou referência possuem maior número de testes raros (esoteric tests) nos tipos de exame ofertados; aqueles em ambientes hospitalares utilizam mais os modos de urgência (tempo de disponibilização de resultados reduzido [STAT]) e os ambulatoriais realizam mais testes denominados de rotina. Hawker et al. ${ }^{(6)}$ demonstraram que exames de rotina em laboratório ambulatorial ou hospitalar são, em sua maioria, testes bioquímicos, em que 35 a 55 tipos de exames compreendem aproximadamente $80 \%$ do volume. Nos laboratórios de apoio ou referência, os 50 tipos mais comuns de exames solicitados abrangem $37 \%$ do volume.

\section{Configuração}

A configuração e o desenho dos equipamentos analíticos em relação à linha de automação são importantes. Destacamos dois dos modelos mais habituais:

- point-in-space - os equipamentos estão conectados diretamente à linha e recebem as amostras, como se fizessem parte dela;

- braços robóticos - braços mecânicos retiram as amostras da linha e encaminham para os equipamentos analíticos.

A escolha entre os dois tipos de configuração deve levar em consideração diferentes parâmetros, como velocidade da linha, menor no primeiro caso descrito devido à menor necessidade de transporte das amostras, tipos de amostra 
(pediátricas, de urina, líquidos biológicos) e a flexibilidade no contexto de manutenção da operação em eventuais paradas da linha, quando se faz necessária a inserção manual das amostras nos equipamentos (estas últimas beneficiadas pelos braços robóticos).

A evolução e a possibilidade de automação das metodologias imunológicas, ou seja, os imunoensaios, permitiram um salto importante na busca pelos benefícios necessários, pois as reações baseadas em ligações antígenos e anticorpos estão presentes em virtualmente todas as áreas da medicina laboratorial(2).

Diferentes aspectos relacionados com os imunoensaios permitiram tal avanço. A utilização de moléculas reveladoras de sinal, introduzidas por meio de ligações competitivas e evoluindo para as reações imunométricas (não competitivas) em substituição aos processos de aglutinação ou precipitação, permitiram, além de mais sensibilidade analítica e velocidade de processamento, a automação dos ensaios com fenômenos imunológicos. Ressaltamos a possibilidade da combinação dessas duas metodologias em equipamentos analíticos únicos, aumentando a quantidade de exames possíveis de serem realizados de forma consolidada e para diferentes tamanhos de moléculas a serem dosadas (competitivos para pequenas moléculas e imunométricos para virtualmente todos os ensaios proteicos) ${ }^{(2)}$.

\section{Fase pós-analítica}

A etapa pós-analítica nas linhas de automação compreende o armazenamento das amostras (soroteca), segundo determinados padrões previamente definidos.

Seu processo de automação (não só o armazenamento, mas a busca de amostras a serem repetidas, diluídas, ou daquelas que necessitam de um teste reflexo) leva a um ganho importante de produtividade e à eliminação de atividades que não geram valor ao produto ou serviço oferecido. Para isso, a utilização de processos de autovalidação dos exames, fornecendo essas diretrizes, é fundamental.

\section{Sistema de informação}

Permeando todas as fases dos processos em medicina laboratorial, o sistema de informação é um dos pontos-chave para o bom funcionamento de uma plataforma de automação.

Em termos gerais, um sistema de informação que administre os processos automatizados (LAS) deve ter, no mínimo, a habilidade $\mathrm{de}^{(8)}$ :

- monitorar a qualidade;

- monitorar os resultados;
- monitorar os equipamentos analíticos e sua operação;

- gerenciar as decisões referentes à repetição de exames;

- gerenciar as decisões referentes aos testes reflexos;

- cancelar testes;

- gerenciar o fluxo de trabalho de todo o laboratório, com base nas necessidades de TAT, capacidade de produção e throughput, modo de utilização do equipamento e disponibilidade para a operação.

Todas essas etapas devem fazer parte da escolha de um sistema de informação laboratorial (LIS) para sua implementação, assim como a validação dos processos. A definição de um LIS deve considerar a adaptação à realidade e aos processos do laboratório.

\section{Modelos de automação disponíveis na medicina laboratorial}

A implementação de um processo de automação laboratorial deve levar em consideração o posicionamento estratégico da empresa e sua forma de atuação. Diferentes modelos de processos automatizados funcionam para diversos negócios, definidos pelos tipos de exame e serviços oferecidos, volume de processamento, atributos estratégicos necessários, capacidade de investimento etc.

Os processos automatizados em medicina laboratorial podem ser descritos nos seguintes grupos:

- stand alone automation (SAA) - em termos gerais, a implementação de um processo automatizado demanda tempo, planejamento e investimentos. O modelo de SAA é uma alternativa interessante. É definido como um processo automatizado isolado, como, por exemplo, um equipamento analítico automatizado que opera por bateladas, um processo automatizado de separação de amostras, armazenagem, distribuição e aliquotagem etc. Na maioria das vezes, esse modelo de operação permite que empresas que operam com volumes menores ou que não possuem porte financeiro para maiores investimentos introduzam processos automatizados e gerem benefícios relacionados com a produção $0^{(11)}$. Uma forte tendência nesse tipo de automação está associada aos processos de testes de biologia molecular e testes genéticos;

- modular automation (MA) - um conceito bastante empregado, que derivou daqueles da SAA, é a modularidade, ou seja, a capacidade de integrar 
diferentes fases da automação em tempos diferentes. Essa abordagem apresenta mais flexibilidade tanto na escolha e na definição das plataformas e fases a serem implementadas quanto no tempo, no aprendizado e na utilização de novos conceitos, prevendo novas decisões ou mudanças de modelos de automação $0^{(11)}$. Devido à menor necessidade de investimento desse modelo, em termos gerais, o retorno sobre o investimento realizado costuma ser em menos tempo ${ }^{(13)}$. Atualmente, as plataformas modulares permitem a integração dos processos pré-analíticos aos analíticos e apresentam, na maioria das vezes, uma vantagem importante na grande quantidade de ensaios disponíveis quando comparadas, por exemplo, ao total laboratory automation (TLA) ${ }^{(11)}$. Um ponto-chave do sucesso da MA é a consolidação de metodologias distintas em únicas plataformas, reduzindo o número de equipamentos que necessitam ser implementados e, consequentemente, trazendo benefícios em termos de velocidade, quantidade de amostras coletadas e atividades manuais que não agregam valor ao produto, como o transporte das amostras entre plataformas ${ }^{(13)}$;

- task target automation (TTA) - esse modelo de automação, direcionada a atividades, tem seu foco principal em suportar atividades específicas dos processos laboratoriais, como as fases pré- e pós-analítica, que possuem alto grau de trabalho pessoal, riscos biológicos e alto potencial de er$\operatorname{ros}^{(5,11)}$. A TTA foi desenvolvida para as atividades de recepção de amostras, aliquotagem, distribuição, retirada de tampas e armazenamento, ou seja, fundamentalmente ela opera na geração de amostras secundárias e fases pré- e pós-analíticas. Destacamos os benefícios desse modelo de automação para laboratórios que possuem grande quantidade de processamento com número diversificado de tipos de amostra e tubos, que necessitam de uma quantidade significativa de alíquotas para ensaios manuais e que realizam grande número de tipos de ensaio e metodologia, como os laboratórios que atuam como apoio a outras empresas. A introdução de um processo automatizado na fase pré-analítica de um laboratório pode representar reduções significativas de custos de até $60 \%$ a $70 \%$ dos gastos totais do laboratório(13);

- total laboratory automation (TLA) - a implementação dos modelos de TLA iniciou-se no Japão e expandiu- se para a América do Norte na década de 1990. O conceito de TLA envolve a automação de todos os processos envolvidos na medicina laboratorial, ou seja, fases pré-, pós- e analítica. Em termos gerais, essas plataformas funcionam com a integração de equipamentos analíticos de diferentes tipos e metodologias em células de trabalho (workcell), conectados por uma esteira (track), como as áreas de bioquímica, sorologia e endocrinologia, hematologia e coagulação. Os processos automatizados na TLA incluem identificação das amostras, centrifugação, aliquotagem, separação e distribuição (sorter), transporte, retirada de tampas, introdução e remoção das amostras nos equipamentos analíticos, armazenamento (soroteca), busca de amostras para repetição de exames e geração de amostras secundárias, além de descarte das mesmas. Toda a movimentação das amostras nas diferentes direções da esteira é gerenciada por meio de leituras por código de barras ou RFID ${ }^{(12)}$. Uma importante vantagem da TLA é a possibilidade de operar com diferentes tipos de amostras, como urina e fluidos biológicos, além de amostras séricas ${ }^{(12)}$.

\section{Discussão}

O desenho das plataformas de automação disponíveis está relacionado com o conhecimento das indústrias que o desenvolvem e deve seguir uma filosofia baseada na relação do laboratório com a atenção à saúde, no fluxo dos processos do laboratório e no negócio com o qual este está envolvido( ${ }^{(8)}$.

Nos EUA, 32\% dos laboratórios possuem algum tipo de automação, sendo que $17 \%$ deles optaram por um modelo de automação total (TLA) ${ }^{(9)}$.

Os fatores que impactam na decisão de investimento em um processo automatizado e no modelo a ser escolhido são os tipos de testes realizados (alinhado ao modelo de negócio da empresa), a quantidade de exames processados e o planejamento futuro da empresa ${ }^{(13)}$.

Os benefícios gerados na introdução da automação são bastante distintos e significativos. A redução de custos operacionais gerada demonstra um retorno sobre o investimento realizado variando de 2,5 a 4,9 anos ${ }^{(7)}$.

A minimização de erros em medicina laboratorial apresenta um impacto significativo na assistência à saúde. Estima-se que cerca de 98 mil norte-americanos morram 
devido aos erros médicos ${ }^{(14)}$. Hawker et al..$^{(7)}$ demonstraram uma redução de $58 \%$ no número de amostras desaparecidas após a implementação do processo de automação, além de redução significativa no TAT e no número de amostras presentes no percentil 95 da meta colocada.

Inúmeros artigos da literatura científica demonstram os projetos realizados na escolha e na implementação de uma plataforma automatizada. A organização do projeto é um ponto fundamental para que o sucesso planejado seja alcançado. O mapeamento dos processos, o planejamento, a participação dos colaboradores, as avaliações contínuas e o acompanhamento dos riscos são partes fundamentais desse processo ${ }^{(10)}$.

\section{Conclusão}

O processo de implementação de um modelo de automação laboratorial gera benefícios importantes para a instituição e, principalmente, para maior segurança na assistência à saúde. A medicina laboratorial possui papel fundamental na cadeia de saúde e, cada vez mais, é fonte geradora de informação com impacto na decisão clínica.

O modelo de automação escolhido deve ser o que meIhor se alinha à estratégia da instituição, não só em termos de investimentos, mas nas escolhas conflitantes (trade offs) na busca pelos benefícios esperados.

A implementação de processos automatizados em laboratórios clínicos deve se manter e pode ser considerada uma tendência para os próximos anos, visto principalmente pelas diferentes possibilidades dessas plataformas para os diferentes portes e necessidades das empresas. $O$ segredo está na escolha do melhor modelo, com base na realidade do laboratório e com o objetivo de reduzir gastos e aumentar a qualidade e a objetividade dos serviços, agregando cada vez mais valor ao cliente.

A Tabela 2 mostra um resumo dos benefícios de um processo de automação em medicina laboratorial.

Tabela 2 Benefícios da automação em medicinal laboratorial

\begin{tabular}{|c|c|c|}
\hline Área de impacto & Benefício & Motivo \\
\hline Assistência à saúde & Maior segurança para o paciente & $\begin{array}{l}\text { - Minimização de erros } \\
\text { - Maior velocidade de entrega de resultados }\end{array}$ \\
\hline Mercado e clientes & Superar/atender às expectativas & $\begin{array}{l}\text { - Maior velocidade de entrega de resultados } \\
\text { - Menor quantidade de novas coletas por erros }\end{array}$ \\
\hline Operação & Redução de custos operacionais & $\begin{array}{l}\text { - Maior produtividade pessoal } \\
\text { - Menos atividades com pouco valor } \\
\text { - Maior velocidade de produção } \\
\text { - Melhor utilização da capacidade instalada } \\
\text { - Padronização dos processos } \\
\text { - Menos documentos } \\
\text { - Melhor utilização do conhecimento pessoal } \\
\text { - Redução de materiais indiretos (tubos) } \\
\text { - Otimização de controles e calibradores } \\
\text { - Otimização de reagentes } \\
\text { - Maior segurança dos colaboradores }\end{array}$ \\
\hline
\end{tabular}




\section{Referências}

1. AKAN, O. A. et al. Evaluation of preanalytic errors in clinical laboratory practice. LabMedicine, v. 37, n. 8, p. 478-80, 2006.

2. BOCK, J. L. The new era of automated immunoassay. Am J Clin Pathol, v. 113, p. 628-46, 2000.

3. BONINI, P. et al. Errors in laboratory medicine. Clin Chem, v. 48, n. 5, p. 691-8, 2002.

4. BOYD, J. C.; FELDER, R. A.; SAVORY, J. Robotics and the changing face of the clinical laboratory. Clin Chem, v. 42, n. 12, p. 1901-10, 1996.

5. DEMIRIS, C.; CIMENT, P. Cost justifying clinical laboratory automation: a task target automation (TTA) approach to justifying laboratory automation. JALA, v. 53, n. 3, p. 20-3, 2000.

6. HAWKER, C. D. et al. Automated transport and sorting system in a large reference laboratory: part 1. Evaluation of needs and alternatives and development of a plan. Clin Chem, v. 48, n. 10, p. 1751-60, 2002.

7. HAWKER, C. D. et al. Automated transport and sorting system in a large reference laboratory: part 2. Implementation of the system and performance measures over three years. Clin Chem, v. 48, n. 10, p. 1761-7, 2002
8. MARKIN, R. S.; SCOTT, A. W. Laboratory automation: trajectory, techonology and tatics. Clin Chem, v. 46, n. 5, p. 764-71, 2000.

9. MELANSON, S. E. F. et al. Selecting automation for the clinical chemistry laboratory. Arch Pathol Lab Med, v. 131, p. 1063-9, 2007.

10. MIDDLETON, S. R. Developing an automation concept that is right for your laboratory. Clin Chem, v. 46, n. 5, p. 757-63, 2000.

11. ORSULAK, P. J. Stand alone automated solutions can enhance laboratory operations. Clin Chem, v. 46, n. 5 , p. 778-83, 2000.

12. PECK-PALMER, O. M. Total lab automation takes teamworks. Med Lab Observer. 2009. Disponível em: $<$ www.mlo-online.com>.

13. SEABERG, R. S; STALLONE, R. O; STATLAND, B. E. The role of total laboratory automation in a consolidated laboratory network. Clin Chem, v. 46, n. 5, p. 751-6, 2000.

14. SPEAR, S. J. Fixing healthcare from the inside. Harvard Business Review, v. 83, n. 9, p. 78-91, 2005. 